\title{
Two-photon excitation with pico-second fluorescence lifetime imaging to detect nuclear association of flavanols
}

Irene Mueller-Harvey a, ${ }^{\mathrm{a}}$, Walter Feucht ${ }^{\mathrm{b}}$, Juergen Polster ${ }^{\mathrm{c}}$, Lucie Trnková ${ }^{\mathrm{d}}$, Pierre Burgos ${ }^{\mathrm{e}}$, Anthony W. Parker ${ }^{\mathrm{e}}$, Stanley W. Botchway ${ }^{\mathrm{e}}$

${ }^{a}$ Chemistry \& Biochemistry Laboratory, Food Production \& Quality Research Division, School of Agriculture, Policy \& Development, University of Reading, P O Box 236, Reading RG6 6AT, UK

${ }^{b}$ Department of Plant Sciences, Technical University of Munich (TUM) Wissenschaftszentrum Weihenstephan (WZW), D-85354 Freising, Germany

${ }^{3}$ Department of Physical Biochemistry, Technical University of Munich (TUM), Wissenschaftszentrum Weihenstephan (WZW), D-85354 Freising, Germany

${ }^{4}$ University of Hradec Králové, Faculty of Science, Department of Chemistry, Rokitanského 62, 50003 Hradec Králové, Czech Republic

${ }^{5}$ Central Laser Facility, Research Complex at Harwell, Science and Technology Facilities Council, Rutherford Appleton Laboratory, Harwell - Oxford, Didcot, OX11 0QX, UK

Corresponding author. Tel.: +44 (0)118 378 6619; fax: +44 (0)118 9352421 .

E-mail address: i.mueller-harvey@reading.ac.uk;

\section{E-mail addresses of co-authors:}

Walter Feucht:

Juergen Polster:

Lucie Trnková:

Pierre Burgos:

Anthony W. Parker:

Stanley W. Botchway: walter.feucht@gmail.com

j.polster@wzw.tum.de

lucie.trnkova@uhk.cz

pierre.burgos@stfc.ac.uk

tony.parker@stfc.ac.uk

stan.botchway@stfc.ac.uk

Keywords: Fluorescence lifetime imaging microscopy, flavanols, epigallocatechin gallate, nuclear binding, histone proteins, multiphoton excitation. 


\section{Two-photon excitation with pico-second fluorescence lifetime imaging to detect nuclear association of flavanols}

1

2Irene Mueller-Harvey ${ }^{\mathrm{a},{ }^{*}}$, Walter Feucht ${ }^{\mathrm{b}}$, Juergen Polster ${ }^{\mathrm{c}}$, Lucie Trnková ${ }^{\mathrm{d}}$, Pierre Burgos

$3^{\mathrm{e}}$, Anthony W. Parker ${ }^{\mathrm{e}}$, Stanley W. Botchway ${ }^{\mathrm{e}}$

4

5 Chemistry \& Biochemistry Laboratory, Food Production \& Quality Research Division, 6School of Agriculture, Policy \& Development, University of Reading, P O Box 236,

7Reading RG6 6AT, UK

$8^{\mathrm{b}}$ Department of Plant Sciences, Technical University of Munich (TUM),

9Wissenschaftszentrum Weihenstephan (WZW), D-85354 Freising, Germany

10 Department of Physical Biochemistry, Technical University of Munich (TUM),

11Wissenschaftszentrum Weihenstephan (WZW), D-85354 Freising, Germany

$12^{\mathrm{d}}$ University of Hradec Králové, Faculty of Science, Department of Chemistry, Rokitanského

1362, 50003 Hradec Králové, Czech Republic

$14^{\mathrm{e}}$ Central Laser Facility, Research Complex at Harwell, Science and Technology Facilities

15Council, Rutherford Appleton Laboratory, Harwell - Oxford, Didcot, Oxfordshire, OX11

160QX, UK

17

18ABSTRACT

19Two-photon excitation enabled for the first time the observation and measurement of

20excited state fluorescence lifetimes from three flavanols in solution, which were $\sim 1.0 \mathrm{~ns}$

21 for catechin and epicatechin, but $<45$ ps for epigallocatechin gallate (EGCG). The shorter 
22lifetime for EGCG is in line with a lower fluorescence quantum yield of 0.003 compared to 23catechin (0.015) and epicatechin (0.018).

\section{4}

25In vivo experiments with onion cells demonstrated that tryptophan and quercetin, which 26tend to be major contributors of background fluorescence in plant cells, have sufficiently 27low cross sections for two-photon excitation at $630 \mathrm{~nm}$ and therefore do not interfere with 28detection of externally added or endogenous flavanols in Allium cepa or Taxus baccata 29cells. Applying two-photon excitation to flavanols enabled 3-D fluorescence lifetime 30imaging microscopy and showed that added EGCG penetrated the whole nucleus of onion 31cells. Interestingly, EGCG and catechin showed different lifetime behaviour when bound to 32the nucleus: EGCG lifetime increased from <45 to 200 ps, whilst catechin lifetime 33decreased from 1.0 ns to 500 ps. Semi-quantitative measurements revealed that the relative 34ratios of EGCG concentrations in nucleoli associated vesicles : nucleus : cytoplasm were 35ca. 100:10:1.

\section{6}

37Solution experiments with catechin, epicatechin and histone proteins provided preliminary 38evidence, via the appearance of a second lifetime ( $\tau_{2}=1.9$ to $\left.3.1 \mathrm{~ns}\right)$, that both flavanols 39may be interacting with histone proteins. We conclude that there is significant nuclear 40absorption of flavanols. This advanced imaging using two-photon excitation and 41biophysical techniques described here will prove valuable for probing the intracellular 42trafficking and functions of flavanols, such as EGCG, which is the major flavanol of green 43tea. 
45Keywords: Fluorescence lifetime imaging microscopy, flavanols, epigallocatechin gallate, 46nuclear association, histone proteins, multiphoton.

47

\section{Introduction}

49Plants synthesise $>4000$ different flavonoid compounds, which can be grouped into several 50different subgroups. Flavanols (Fig. 1) are an important subgroup that is widespread in 51plants and plant foods [1]; they are also precursors of condensed tannins, which are the 52fourth largest group of natural plant products after cellulose, hemicellulose, and lignin [2]. 53These polyphenolic compounds are attracting considerable interest, because diets rich in 54fruits and vegetables are associated with improved health and a reduction of age-related 55diseases such as cancer, osteoporosis and cardiovascular diseases [3-7]. Flavonoids are 56considered to be 'lifespan essentials' and recent reviews suggest that their antioxidant 57properties alone are unlikely to explain their beneficial effects on human health or their 58functions in plants [8-10].

59

60A consensus is emerging that in vitro and in vivo experiments need to probe the 61bioavailability of these polyphenols and their molecular targets [3,6,8,10-11]. In vitro 62studies have tended to require 10 to 100 -fold higher polyphenol concentrations than are 63usually found in mammalian plasma and tissues in order to achieve many of the reported 64medicinal effects [5,12]. However, the existence of high-affinity targets for dietary 65polyphenols might explain their health-promoting effects and in this context it is pertinent 66to examine more closely recent evidence that nuclei from both plant and mammalian cells 67acted as sinks for flavanols [13-17]. Although the function of these secondary plant 
68metabolites requires further elucidation, evidence is emerging that they may be important in 69cell development. For example, loss of flavanols has been linked to defective pollen 70development [14]. Different types of flavanol distribution patterns were observed in Tsuga 71canadensis at the sub-nuclear level [13] and the authors questioned whether the epigenetic 72code of histones could affect flavanol-chromatin associations. Moreover, Feucht et al. [15] 73found identical flavanol patterns within different cell lineages in a meristematic plant tissue 74and suggested that this could be indicative of a synchronized, transcriptional regulation. In 75addition, nuclear flavanol concentrations clearly depended on the season, i.e. during 76dormancy they were almost absent but during growth periods relatively high amounts were 77observed [18]. The fact that flavanols were associated with both interphase and mitotic 78chromosome states posed the question of whether flavanols might be associated with 79histones. If this is the case, then this could open a new perspective on genomic regulation. 80

81This research by Feucht's group made use of the fact that flavanols form a blue 82condensation product with dimethylaminocinnamaldehyde (DMACA) [19]. The DMACA 83reagent is, however, a relatively aggressive reagent that requires $0.75 \mathrm{M}$ sulfuric acid for 84the staining reaction and this could cause some physical damage within the cells. Polster et 85al. [18], therefore, tested the existence of nucleus-bound flavanols with a milder technique, 86i.e. laser microdissection and pressure catapulting (LMPC), which separated intact nuclei 87 from cells and these also stained blue in the subsequent DMACA reaction. Nevertheless, 88LMPC causes physical rupture of the cytoplasm that surrounds the nuclei and could have 89given rise to an artificial DMACA reaction. Moreover, histological studies with DMACA 90cannot distinguish between different flavanols or between flavanol monomers, oligomers or 
91polymers [19]. Techniques are therefore required that can establish the sub-cellular

92localisation, and concentrations therein, of flavanols to probe their functionality and 93metabolism in plant and mammalian cells.

\section{4}

95Nifli et al. [29] recently applied confocal fluorescence microscopy to map the intracellular 96distribution of a major plant flavonol, i.e. quercetin (Fig. 1), which has a UV absorption $97 \lambda_{\max }$ of $372 \mathrm{~nm}$. Quercetin revealed a specific fluorescence (488 $\mathrm{nm}_{\mathrm{ex}} / 500-540 \mathrm{~nm}$ em $)$ in the 98cellular environment at physiologically relevant concentrations $(<5 \mu \mathrm{M})$, which the authors 99attributed to non-covalent binding to cellular components. Intracellular tracing of flavanols $100\left(\lambda_{\max } \sim 280\right.$ nm; Fig. 1 and Fig. S1) by UV-Vis spectroscopy or confocal fluorescence 101microscopy is, however, not possible because plant and mammalian cells contain numerous 102compounds which would interfere with the detection of flavanols by giving background 103fluorescence signals (termed “auto-fluorescence”). Fig. 2 illustrates the photophysical 104processes in a conventional Jablonski diagramme, which depicts one- and two-photon 105excitation and various relaxation pathways that are open to the electronic excited state 106following photon(s) absorption.

108In fluorescence life-time imaging microscopy (FLIM) both fluorescence intensities and 109fluorescence lifetimes of specific compounds can be measured at each pixel in the image $110[21,22]$. In addition, variations in fluorescence lifetime can provide further image contrast: 111lifetime shifts can serve as sensitive probes for detecting molecular interactions and may 112yield information on a compound's environment, such as $\mathrm{pH}$ or oxygen concentration [2311325]. Lifetime, $\tau$, is derived from the time-constant of the fluorescence decay (Fig. 2), where 
$114 \tau=1 / \mathrm{k}_{\text {fluorescence }}$ FLIM is based on either single- or multi-photon excitation techniques.

115Multi-photon excitation with femtosecond lasers offers many advantages for biological

116measurements over more conventional single photon excitation [23,26]:

$117 \checkmark$ Excitation with red light that is not directly absorbed by cellular

118 materials.

$119 \checkmark$ Reduced cellular toxicity in biological studies.

$120 \checkmark$ Reduced photo-bleaching.

$121 \checkmark$ Deeper penetration of the near-infrared light into the biological

122 specimen.

$123 \checkmark$ Femtolitre volume excitation.

$124 \checkmark$ A flexible imaging platform that is capable of resolving several

125 (and related) compounds.

126The ability to deliver UV-equivalent photon energies directly beneath UV 127absorbing materials and molecules.

128An ability to perform time-resolved studies due to the short pulsed light

129source.

130In two-photon excitation (2PE) the simultaneous absorption of two lower energy photons

131mimics the absorption of a single photon of equivalent higher energy (Fig. 2). Thus, 2PE at $132560 \mathrm{~nm}$ mimics UV excitation at $280 \mathrm{~nm}$ [23,27]. In FLIM ultrafast lasers providing pulse 133lengths of the order of 200 femtoseconds $\left(200 \times 10^{-15} \mathrm{~s}\right)$ enable time-resolved 134measurements, which can detect molecular interactions in solution and cells [23-25,28] and 
135can be used to construct fluorescence life-time maps of a compound's distribution within 136viable cells.

138Here we describe 2PE experiments designed to eliminate any doubts regarding the results 139from previous histological studies that employed the DMACA staining reagent. Two140photon excitation coupled to 3-D fluorescence lifetime imaging microscopy enabled 141examination of intact biological tissues and highly localised, non-destructive and selective 142detection of flavanols. The fluorescence behaviour of three flavanols, catechin, epicatechin 143and epigallocatechin gallate (EGCG) (Fig. 1), was measured first in model solution systems 144and then in two natural cell systems, onion epidermis cells and Taxus pollen mother cells. 145These flavanols were chosen because they are widespread in plants and are also 146bioavailable and bioactive in several in vitro and in vivo mammalian cell systems 147[1,6,9,29]. Solution phase studies were first used to optimise and measure spectroscopic 148shifts and lifetime changes of free flavanols versus flavanols bound to DNA or histone 149proteins at normal physiological $\mathrm{pH}$ values. The optimised spectroscopic parameters were 150then applied to probe the intra-cellular location of externally added flavanols in Allium 151cepa cells and of endogenous flavanols in Taxus baccata cells. The same plant models had 152been tested previously with the DMACA staining reagent $[14,30]$.

153

1542. Methods and materials

1552.1. Reagents

156The following reagents were purchased from Sigma-Aldrich Company Ltd, UK: 
157(+)-catechin (98\%), (-)-epicatechin (90\%), (-)-epigallocatechin gallate (95\%; EGCG), tris158(hydroxymethyl)amino methane (Tris), $\mathrm{K}_{2} \mathrm{HPO}_{4}$ (ACS reagent, ( $\geq 98 \%$ ), $\mathrm{KH}_{2} \mathrm{PO}_{4}$ (ACS 159reagent, ( $\geq 99 \%)$, DNA from calf thymus and Histone type II-A. Histone was supplied by 160Roche Diagnostics Ltd, UK. Histone sulphate from calf thymus was purchased from Fluka 161(Sigma-Aldrich Chemie, Steinheim, Germany; Polster et al., 2003). Ethanol (LiChrosolv, $\geq$ 16299.9\%) was purchased from VWR-Merck, UK.

163

164Tris buffers $(0.1 \mathrm{M})$ were prepared and adjusted to $\mathrm{pH} 7.0$ and 8.0 with $\mathrm{HCl}$ and phosphate 165buffers $(0.1 \mathrm{M})$ at $\mathrm{pH}$ 5.8, 7.1 and 8.2 were prepared using $\mathrm{K}_{2} \mathrm{HPO}_{4}$ and $\mathrm{KH}_{2} \mathrm{PO}_{4}$ as 166described [16].

167

1682.2. Calculation of relative fluorescence quantum yields of the flavanols 169Flavanols were dissolved in methanol to yield 0.01 M stock solutions. Subsequent dilutions 170 for 20 and $40 \mu \mathrm{M}$ flavanol concentrations were made with sodium phosphate buffer (pH 1717.4, 0.1 M, 0.05\% sodium azide). These were placed in a $10 \mathrm{~mm}$ quartz Suprasil 172fluorescence cuvette (Hellma, Germany) and UV-Vis spectra were first recorded from 190 173to $500 \mathrm{~nm}$ using a Helios $\beta$ spectrophotometer (Spectronic Unicam, U.K.). Then 174fluorescence spectra were recorded using a luminescence LS-55 spectrometer (Perkin 175Elmer, U.K.) from 290 to $530 \mathrm{~nm}$ with excitation at $295 \mathrm{~nm}$ under continuous stirring. The 176excitation and emission slits were both set to $5 \mathrm{~nm}$ and scanning speed was $200 \mathrm{~nm} \min ^{-1}$. 177All experiments were carried out at $37^{\circ} \mathrm{C}$. The literature reported a quantum yield of 0.12 178for tryptophan (Trp) at $270 \mathrm{~nm}$ (website) and we confirmed this for $295 \mathrm{~nm}$. Therefore, the 179quantum yields of flavanols (Flav) were calculated relative to tryptophan using the 
180integrated area between 300 and $530 \mathrm{~nm}$ under the fluorescence spectra [31-33] according 181to:

1862.3. Flavanol solutions

187Flavanols were dissolved in ethanol $(\sim 10 \mathrm{mM})$ and prepared fresh on a daily basis. Just 188before measuring the fluorescence lifetimes, aliquots $(10 \mu \mathrm{L})$ were removed and diluted 189with buffer, DNA or histone protein solutions (90 and $40 \mu \mathrm{L}$ ) to obtain flavanol 190concentrations between 1 and $2 \mathrm{mM}$.

191

192DNA (0.6 mg) was dissolved in Tris buffer (pH 8.0; $30 \mathrm{~mL})$ overnight at $4{ }^{\circ} \mathrm{C}$. Sigma 193histone (5.9 mg) was dissolved in Tris buffer (pH 7.0 and 8.0; $2.85 \mathrm{~mL})$. Ethanol (98 $\mu \mathrm{L})$ 194was added to the $\mathrm{pH} 8.0$ buffer to facilitate dissolution. Histone sulphate (0.8 $\mathrm{mg}$ ) was 195dissolved in Tris buffer (pH 7.0 and 8.0) according to Polster et al. [16]. The supernatants 196were used after centrifugation. Roche histone $(1.0 \mathrm{mg})$ was dissolved in Tris buffer (pH 7.0 197and 8.0; $500 \mu \mathrm{L})$ and ethanol $(10 \mu \mathrm{L})$.

198

1992.4. Plant samples

200The thin adaxial epidermis from onion (Allium cepa) bulb scale was removed, cut into 2 $201 \mathrm{~cm}^{2}$ pieces and incubated with aqueous catechin or EGCG solutions (1 mM; $\left.20 \mathrm{~mL}\right)$ for up 202to 8 h [30]. 
204Male cones from yew (Taxus baccata) were harvested on $5^{\text {th }}$ October 2008. The eight cover 205leaves were removed and the yellow anthers were gently squeezed with tweezers in order to 206release the mother pollen cells. Preliminary experiments revealed that these cells stained 207dark blue with the DMACA reagent (10 mg DMACA dissolved in $1 \mathrm{~mL}$ of $0.75 \mathrm{M} \mathrm{H}_{2} \mathrm{SO}_{4}$ ) 208[30].

209

\subsection{Multiphoton microscopy}

211The set up used in this study has been previously described [23]. Briefly, a custom built 212two-photon microscope was constructed using scanning XY galvanometers (GSI Lumonics 213Ltd). A diode-pumped (Verdi V18) titanium sapphire (Mira F900) operating at 700-980 nm 214generated laser light at a wavelength of $585 \pm 2 \mathrm{~nm}$ and was used for the solution studies 215and at $630 \pm 2 \mathrm{~nm}$ for the plant cell studies through an optical parametric oscillator (OPO, 216APE-Coherent GmbH, Berlin, Germany) operating at 180 fs pulses at $75 \mathrm{MHz}$. The pulse 217width was maintained using a femto control unit (APE Coherent GmbH). The laser beam 218was focused to a diffraction-limited spot using a water-immersion ultraviolet corrected 219objective (Nikon VC x60, NA 1.2) and specimens were illuminated at the microscope stage 220of a modified Nikon TE2000-U with UV transmitting optics. Fluorescence emission was 221collected without descanning, bypassing the scanning system, and passed through a $340 \pm$ 22220 nm interference filter (U340, Comar Instruments, Cambridge, UK). Emission 223fluorescence was detected using an external fast microchannel plate photomultiplier tube 224(Hamamatus R3809U-50) and recorded using a Time-Correlated Single Photon Counting 225(TCSPC) PC module SPC830 (Becker and Hickl GmbH, Berlin, Germany). Fluorescence 
226lifetime image microscopy was performed by synchronising the XY galvanometer positions 227with the fluorescence decay. The X,Y galvanometers were raster scanned at $1 \mathrm{~ms}$ or $2 \mathrm{~ms}$ 228per pixel for 128 x 128 or 256 x 256 image size, respectively, giving a 33 sec per image 229frame. The presented images were three accumulations to allow for enough photon counts 230per channel for the data analysis.

231

2322.6. Image analysis

233Steady state grey scale images (8 bit, up to 256 x 256 pixels) are produced by binning all 234decay photons as a single channel. Fluorescence lifetime images were obtained for control 235cells and flavanol-loaded cells by analysing the decay at individual pixels using a single or 236double exponential curve fitting (SPCImage 2.94 analysis software Becker and Hickl). A 237thresholding function within the FLIM analysis software ensured that noncorrelating 238photons leading to background noise arriving at the detector were not included in the 239analysis. Single point decay analysis was carried out without binning while FLIM was 240analysed with a maximum of 2 binning.

\section{1}

\section{Results and discussion}

\subsection{Flavanol fluorescence lifetimes in aqueous solutions}

244It is well known that flavanols oxidise readily in alkaline $\mathrm{pH}$ [4], therefore lifetimes were 245first examined at $\mathrm{pH}$ values ranging from 5.8 - 8.2. Fig. 3 shows that the fluorescence 246lifetime, $\tau$, of catechin (2 mM catechin solution in $0.1 \mathrm{M}$ phosphate buffer) was relatively 247stable between pH 5.8 and 7.1: $\tau$ was 1.0 ns at the start and 0.9 ns after 20 min. However, at 248pH 8.2 the lifetime changed from 1.0 to 0.7 ns within 20 minutes. When the same 
249measurements were conducted under a nitrogen blanket, lifetime reduction was kept to 9\% 250over a 30 min period and this agrees with Sang et al. [34] who found that flavanols were 251not oxidised under nitrogen. Therefore, all subsequent solution measurements were 252determined immediately after mixing the solutions, i.e. within 30 seconds. Fig. 3 also 253shows that $\mathrm{pH}$ per se had no effect on catechin lifetimes: $\tau$ of catechin was $~ 1.0 \mathrm{~ns}$ at $\mathrm{pH}$

2545.8, 7.1 and 8.2. The reduction in $\tau$ values can also not be ascribed to sample concentration 255or the presence of non-interacting or energy transfer products, as the excited state lifetime 256is independent of both of these.

257

258The natural lifetime of catechin in solution in the absence of oxygen is $~ 1.1$ ns (Fig. 3). 259This reduces, via quenching, as expected in the presence of dissolved oxygen $\left(7.6 \mathrm{mg} \cdot \mathrm{L}^{-1}\right)$ 260at room temperature and pressure to 1 ns. It is worth noting that at high oxygen 261concentrations, $30 \mathrm{mM}$, the quenched lifetime observed will be as expected taking into 262account diffusion control rate. Therefore the subsequent change in lifetime (Fig. 3) (to 0.7 263ns after 20 min in oxygen) is very likely due to the formation of a deprotonated or oxidised 264product as the $\mathrm{OH}$ groups in the B-ring are particularly susceptible to deprotonation and 265therefore oxidation at alkaline $\mathrm{pH}$ [35]. It is interesting to note that the reduced lifetime 266fitted well to a single exponential decay, again indicating a single fluorescent molecular 267species is present and favouring the observed decreases in lifetime results from either a 268photoproduct, which also fluoresces, or an oxygen quenched process. Further studies using 269high performance liquid chromatography may help identify these oxidised products. 
271Importantly, Fig. 4 shows that flavanols had different fluorescent decay curves.

272Fluorescence lifetimes of catechin and epicatechin were similar (1.0 and $1.1 \mathrm{ns,}$ 273respectively). However, in the case of EGCG at pH 8.1 (2 mM flavanol solutions in $0.1 \mathrm{M}$ 274phosphate buffer) the lifetime was found to be within the instrument response function and 275Fig. 4 shows only the characteristics of the fast micro-channel plate ( $<45 \mathrm{ps})$. Utrafast time276resolved Kerr gated fluorescence spectroscopy will be needed to resolve the EGCG lifetime 277in the future. EGCG differs from catechin and epicatechin by the presence of a galloyl 278group at C-3 (Fig. 1). The lifetime of the excited state is given by the sum of the different 279competing relaxation processes, which include fluorescence, non-radiative decay, 280intersystem crossing and chemical reaction as illustrated in Fig. 2. The shorter lifetime for 281EGCG is most likely due to the presence of additional phenolic groups, which would be 282expected to enhance the solvation effects and which in turn would influence the non283radiative decay processes. These extra phenolic groups also enhance its antioxidant 284properties [36] and this presumably makes it more susceptible to oxidation. Indeed, the 285fluorescence quantum yield of EGCG is much lower than that of catechin or epicatechin 286(Table 1) suggesting that the non-radiative rate ( $\mathrm{k}_{\mathrm{IVR}}$; Fig. 2$)$ dominates in the relaxation of 287the electronic excited state.

288

289At pH 8, epicatechin also had a two-component fluorescence decay lifetime (see footnote in 290Supplementary Table). The exact physical origin of the bi-exponential lifetime is unknown. 291However, it is not uncommon for fluorophores in complex cellular environments to 292demonstrate multiple decay times as seen in Table 2. Different decay times represent 293differing physical influences that the nascent electronic excited states are subjected to and 
294consequently may lead to differences in the efficiency of the energy loss process and return 295to the ground state. The fact that we see a bi-exponential decay indicates that the flavanols 296find themselves in two differing states and/or two different environments; for example free 297and bound forms (Supplementary Table). Further investigations studying the ultrafast 298dynamics will be needed to help explain these differences and/or whether diastereoisomers 299such as catechin and epicatechin have different fluorescence properties.

3013.2. Fluorescence lifetime imaging microscopy

3023.2.1. Control experiments with onion cells

303The experimental conditions developed above for flavanol solutions were applied initially

304to onion root cells (tissue soaked in water for $5 \mathrm{~h}$; and followed by two-photon excitation at $305585 \mathrm{~nm}$ ). Lifetime decay curves, at several different points in the cells, could be fitted to a 306single exponential decay giving a $\tau$ value between 2.3 and $2.6 \mathrm{~ns}\left(\mathrm{x}^{2}=1.05\right)$. It is highly 307likely, however, that under these excitation conditions the emission and lifetime values are 308mainly due to auto-fluorescence contributions from tryptophan [23]. In order to avoid 309significant background fluorescence signals from other cellular materials, in particular 310aromatic amino acids, e.g. tryptophan, when using UV excitation at $290 \mathrm{~nm}$ (equivalent to $311580 \mathrm{~nm}$ 2PE excitation) the 2PE excitation wavelength was shifted to $630 \mathrm{~nm}$, which has 312been shown to give little background interference [23]. Control experiments were then 313carried out without added flavanols, i.e. in the presence of just water, in order to 314substantiate that the fluorescence was due to flavanols. The onion sample without added 315flavanol showed only weak auto-fluorescence and a t value of $0.8 \mathrm{~ns}\left(\chi^{2}=1.60\right)$ (Fig. 5c) 316confirming that our FLIM measurements were tracking the flavanol presence in cells (see 
317Section 3.2.2. below). Whole onions are known for their high quercetin concentration (Fig. 3181) [6], but given the low photon count, we can conclude that neither tryptophan nor 319quercetin interfered with flavanol detection, $\lambda_{\mathrm{em}}$, at $340 \pm 20 \mathrm{~nm}$ if $2 \mathrm{PE}$ with $\lambda_{\mathrm{ex}}$ was $630 \mathrm{~nm}$. 320

\subsubsection{Absorption of flavanols by onion nuclei}

322Fig. 5a and 5b show fluorescence lifetime maps of cells in an onion epidermis, which had 323been soaked in $1 \mathrm{mM}$ aqueous flavanol solutions. Following absorption of catechin or 324EGCG, the fluorescing nuclei and several bright, small spots of $\sim 2$ to $7 \mu \mathrm{m}$ were clearly 325visible to a much greater extent than the surrounding cell matrix. Careful analysis of Fig. 5b 326showed a bright spot of $4 \mu \mathrm{m}$ diameter. It is known that inactive nuclei possess very small 327nucleoli of the order of $\sim 1 \mu \mathrm{m}$ [37]. The observed spot is too large to be a nucleolus, we 328therefore propose that the bright spot was a clustering of perinucleolar organiser regions 329(NORs) [38]. NORs tend to surround the nucleoli and strongly absorb flavanols [13].

331This study demonstrated that FLIM combined with 2PE at $630 \mathrm{~nm}$ enabled in vivo detection 332of both catechin and EGCG and avoided interference by tryptophan or quercetin, as the 333control showed hardly any fluorescence (Fig. 5c). We have previously shown that there is 334negligible excitation of cellular auto-fluorescence, particular from tryptophan, following 335multiphoton excitation at $630 \mathrm{~nm}$ [23]. Although tryptophan may be excited by multi336photon treatment at $590 \mathrm{~nm}$, which is equivalent to single photon excitation (1PE) at 295 337nm, this diminishes by a factor of 10 at $630 \mathrm{~nm}$. Furthermore, the excited state lifetime of 338tryptophan ( $3 \mathrm{~ns})$ is significantly different to that of the flavanols investigated here. These 339findings, therefore, provided clear and unequivocal evidence for nuclear flavanol 
340absorption. Since the excited state lifetime may be influenced by the environment of the 341flavanols, the colour trend seen in the FLIM images (Fig. 5a,b) may be due to slight 342differences in the environment of the absorbed flavanols. A series of $z$ axis images taken 343through a cell revealed that EGCG was detectable throughout the nucleus and not just at the 344surface (Video Clip S1). EGCG appeared to be concentrated in the NORs; relative 345proportion of EGCG photon counts were 1 to 3 (cytoplasm) : 10 (nucleus) : 100 to 150 346(NORs) (data not shown).

3483.3. FLIM lifetimes of bound versus free flavanols in solution 349Fluorescence decay curves of nucleus-bound catechin were best fitted to two components, 350i.e. $\tau_{1}=0.5$ ns $(77.5 \%)$ and $\tau_{2}=2.7$ ns $\left(22.5 \% ; \chi^{2}\right.$ of 1.04$)$ (Table 2$)$. It is unlikely that $\tau_{2}$ 351emanates from tryptophan as the same experiments with EGCG fitted to a single 352component decay with an average $\tau$ of $0.25 \pm 0.05$ ns (Table 2). The increase in EGCG 353lifetime from $<0.045 \mathrm{~ns}$ in solution (Fig. 4) to $0.25 \mathrm{~ns}$ in the nucleus is a reverse of the 354trend seen for catechin, which showed a lifetime of $~ 1$ ns in solution and 0.5 ns in the 355nucleus.

356

357The effect of nuclear association generating different lifetimes is given in Table 2 and was 358recorded when Fig. 5 was taken. Taken together, these observations suggest that the two 359flavanols (catechin and EGCG) may differ in their interaction mechanisms with nuclear 360components. The lowering of a lifetime indicates either an enhanced non-radiative decay 361(through for example formation of hydrogen bonds) [31] or possibly self-association which 362has been reported for catechin [39]. A decrease in lifetimes upon cellular absorption has 
363also been reported for 5-hydroxytryptophan and was attributed to self-quenching or 364environmental effects [23]. Further research will be needed to establish whether oxidation 365during the cellular absorption experiment could have contributed to the shorter catechin 366lifetime (Fig. 3) and whether oxidation would have increased EGCG fluorescence lifetime. 367It seems, however, more likely that this contrasting lifetime behaviour is indicative of 368different interaction mechanisms.

369

3703.4. Endogenous flavanols in Taxus baccata

371The same 2PE experimental conditions were then applied to pollen mother cells which had 372been isolated from microspores of male Taxus baccata cones. According to Feucht et al. 373[14] late tetrads and early microspores possess endogenous catechin and epicatechin. We 374observed, however, fluorescence lifetimes, which could be fitted to single component 375decays with $\tau$ of $0.2 \mathrm{~ns}$ and which resembled EGCG, rather than catechin or epicatechin 376(Table 2). Interestingly, the photon count of the signal to noise ratio from endogenous 377flavanols in the Taxus cells was not dis-similar to onion cells soaked in a 1 mM EGCG 378solution. Younger cones at the tip of the Taxus baccata twig yielded twice as many photons 379compared to slightly more mature cones from further along the twig. This finding agrees 380with previous observations $[14,15]$ that nuclear DMACA staining for flavanols was most 381intense during high cell activity, e.g. in mitotic and stem cells. Interestingly, it also 382coincides with observations of higher EGCG concentrations in foetal than maternal plasma 383of rats: absorbed catechins were found in the brain, eye, heart, lung, kidney, liver and 384placenta of fetal organs [40]. 


\subsection{Flavanol fluorescence lifetimes in the presence of DNA or histone proteins}

387The fact that flavanols bind to the nucleus raises an important question: which nuclear 388components act as the binding sites? Several previous studies demonstrated that DNA 389interacts with planar flavonoids, such as flavonols and anthocyanidins, and depending on 390the experimental conditions, these interactions were either weak or led to intercalation [41]. 391However, flavanols are not planar and may therefore not be able to intercalate with DNA. 392We, therefore, explored fluorescence lifetime behaviour of flavanols in the presence of 393DNA. Addition of DNA had no effect on catechin or epicatechin lifetimes in aqueous 394solutions (2 mM; pH 8 in Tris buffer; data not shown). This agrees with results from UV395Vis spectroscopic titrations which also found that DNA did not interact with catechin or 396EGCG in $0.1 \mathrm{M}$ Tris at $\mathrm{pH} 7.4$ or 8.0 [16].

398However, Polster et al. [16] reported that histone proteins might be the nuclear targets for 399catechin and EGCG. Using UV-Vis titration experiments, they showed that both flavanols 400bound to histone sulphate and interactions were more pronounced at $\mathrm{pH} 8.0$ than 7.4 in Tris 401buffer. Since these titration experiments required approximately $1 \mathrm{~h}$ in total [42], it could be 402argued that this might be sufficient time for oxidative reactions and artefact formation to 403occur especially at higher $\mathrm{pH}$ values as determined in Fig. 3. The present fluorescence 404lifetime measurements were, however, made within $30 \mathrm{~s}$ of mixing the flavanol and histone 405solutions (note: the fluorescence lifetime experiments were also done in Tris buffer, as the 406UV-Vis titrations revealed that histone sulphate showed a less pronounced interaction with 407catechin in phosphate than Tris buffer [16]). 
409The lifetimes of catechin and epicatechin in the presence of histone proteins were 410investigated at two concentrations (1.1 and $1.9 \mathrm{mM})$ and $\mathrm{pH}$ (7 and 8) (Supplementary 411Table). Given the short lifetimes recorded and the errors in fitting bi-exponential decays, 412the data for the different flavanols need careful interpretation. At this stage we are unable to 413clearly identify whether or not histones bind to flavanols, which would be expected to be 414shown by a change in lifetime (i.e. due to quenching). From other work it is, however, also 415clear that histone preparations differ in their ability to associate with flavanols [13] and, 416therefore, further fluorescence studies will be needed. Nevertheless, these initial findings 417demonstrate the potential power of studying flavanol-histone interactions by fluorescence 418methods. It is now also evident that much fundamental work is needed for characterising 419how the chemical environment (including $\mathrm{pH}$ and oxygen concentration) influence 420fluorescence lifetimes, quantum yields and spectra of flavanols. With regard to $\mathrm{pH}$, the $\mathrm{pK}_{\mathrm{a}}$ 421values of catechin, for example, are ca. 8.6 and 9.4 [43] and thus at pH 8 three different 422protolyte species exist for catechin: $\mathrm{BH}_{2}, \mathrm{BH}^{-}$, and $\mathrm{B}^{2-}$. The fluorescence behaviour of each 423of these species will need to be understood and only through such careful measurements 424can these types of fluorescence measurements provide the much needed tool for elucidating 425the interactions between flavanols, histones and DNA.

426

4273.6. Possible role of nuclear flavanols beyond an antioxidant function 428Nuclear absorption has been reported not only for flavanols [13] but also for some other 429flavonoids. Arabidopsis thaliana nuclei absorb flavonols [44.45], Drosophila follicle nuclei 430absorb quercetin [46] and Flaveria chloraefolia nuclei absorb sulfonated flavanols [47]. It 431used to be widely accepted that the major function of polyphenols such as flavonoids was 
432to protect DNA against UV damage and oxidative stress, but this has now been questioned $433[8,10]$. Instead they were shown recently also to affect cell signalling and gene expression 434[48,49]. Flavanols are involved in the transcriptional activation of genes and modulation of 435epigenetic changes [36,50].

436

437Whilst several dietary flavonols and the green tea flavanol, EGCG, have been implicated in 438interacting and protecting DNA against damage [41,51, 52], the fact that they inhibit DNA 439methyltransferases in vitro and in vivo at the $\mu$ molar to sub- $\mu$ molar level is potentially more 440important for their effects on health [5,7,29,48,53]. Moreover, EGCG was also a potent 441inhibitor of histone acetyltransferase [50]. Histone acetylation has previously been shown 442to affect flavanol association $[13,16]$ and is known to alter the chromatin structure, which in 443turn has been linked to the transcriptional activation of genes [15]. Both processes, DNA 444methylation and histone acetylation, are involved in epigenetic changes [54]. Indeed, 445Yamada et al. [53] concluded that EGCG may have inhibitory effects on the epigenetic 446changes that occur during carcinogenesis and aging. Whilst we found no evidence for 447interactions between flavanols and DNA, the results presented here in terms of flavanol 448association and penetration through the nucleus do not rule out the possibility that histones 449may be a target for EGCG, catechin and epicatechin. Solution phase ultrafast structure and 450dynamics studies such as time resolved infra-red (IR) or time resolved 2-dimensional IR 451will be needed to probe the origin of the bi-exponential lifetimes, which differed between 452the three flavanols. Such time resolved spectroscopic techniques will indicate the functional 453groups responsible for the fast dynamics that differ amongst these flavanols. 


\subsection{Future prospects}

456Given the recent discoveries identifying that flavanols may well be involved in epigenetic 457changes, highly sensitive techniques will be needed to trace their uptake and trafficking at 458the sub-cellular and sub-nuclear level and at physiologically relevant concentrations. Our 459results suggest that not all flavanols will interact via the same molecular mechanism and 460this will require new techniques with sufficient specificity and sensitivity. New 461developments in fluorescence lifetime imaging techniques and ultra-fast spectroscopy, as 462demonstrated here, may hold the key and pave the way for studying their functions and 463synthesis in plant cells. The trafficking, uptake and subcellular localisation of flavanols is 464of acute interest also for current research on tannin synthesis in plants [55]. Unravelling this 465last hurdle of flavonoid biosynthesis, storage and release would facilitate the development 466of new plant varieties with tannin compositions that can offer enhanced biological activities 467for nutrition and health [11].

468Such analytical developments will facilitate new types of biological experiments that can 469test how these compounds, when present in plant foods, can impact on mammalian cells 470and health. Although FLIM is a new technique to both mammalian and plant cell biologists 471alike, its application is growing rapidly [23-26,28], particularly in protein-protein 472interactions that involve energy transfer processes. However, this is the first study to report 473FLIM for other plant components and as the technique becomes more readily available, its 474impact can only grow. This study has shown that relatively small changes in flavanol 475structures (EGCG versus catechin or epicatechin; Fig. 1) lead to measurable changes in 476lifetime behaviour in the free and bound states. As plants synthesise several types of 477flavonoids, that vary in oxidation and substitution patterns [1], it is expected that other 
478flavonoid compounds will be detectable using different combinations of excitation and 479emission wavelengths.

480

\section{Conclusions}

482In conclusion, 2-photon excitation at 585 and $630 \mathrm{~nm}$ has enabled for the first time the 483measurement of fluorescence lifetimes from three flavanols, catechin, epicatechin and 484EGCG, in solution and in vivo. Lifetimes ranging from $<45$ ps to 1 ns in solution have been 485determined. In vivo experiments with onion cells demonstrated that tryptophan and 486 quercetin have sufficiently low absorbance at $630 \mathrm{~nm}$ and this allowed the detection of 487externally added and endogenous flavanols within Allium cepa and Taxus baccata cells. 488Interestingly, fluorescence decay curves of catechin and EGCG differed markedly both in 489solution and when bound at the nucleus. This fact could be used in the future for selectively 490tracing the different flavanols in vivo. Furthermore, this work demonstrates how the 491application of fluorescence lifetime technology may be used to investigate the way 492flavanols interact with individual cellular components. We also conclude that flavanols are 493absorbed by cell nuclei and this provides new research challenges with regard to their 494intracellular functions.

495

496Semi-quantitative measurements revealed that the relative ratios of EGCG concentrations in 497perinucleolar organiser regions : nucleus : cytoplasm were approximately 100:10:1.

498Moreover, 3-D FLIM showed that externally added EGCG penetrated the whole nucleus of 499onion cells and was not just absorbed on the surface. The FLIM technique described here 500proved therefore a significant advance to DMACA staining and is capable of providing 
501quantitative biophysical information to probe the intra-cellular functions of flavanols such 502as EGCG, which is the major flavanol of green tea.

503

504Acknowledgements

505We are grateful to the Science and Technology Facilities Council for facility access time

506and financial support (No 81072) and to Professor R.H. Bisby, Salford University, for

507helpful discussions.

508

509References

510[1] J.A.M. Kyle, G.G. Duthie, in: Ø.M. Anderson, K.R. Markham (Eds.), Flavonoids:

511 Chemistry, Biochemistry and Applications, CRC Press, Boca Raton, 2006, pp. 219-

$512 \quad 255$.

513[2] P.J. Hernes, J.I. Hedges, Geochim. Cosmochim. Acta. 68 (2004) 1293-1307.

514[3] R.M. Hackman, J.A. Polagruto, Q.Y. Zhu, B. Sun, H. Fujii, C.L. Keen, Phytochem.

$515 \quad$ Rev. 7 (2008) 195-208.

516[4] J.D. Lambert, R.J. Elias, Arch. Biochem. Biophys. 501 (2010) 65-72.

517[5] S. Sang, J.D. Lambert, C.S. Yang, J. Sci. Food Agric. 86 (2006) 2256-2265.

518[6] G. Williamson, C. Manach, Am. J. Clin. Nutr. 81 (suppl) (2005) 243S-55S.

519[7] W.J.L. Lee, J.-Y. Sim, B.T. Zhu, Mol. Pharmacol. 68 (2005) 1018-1030.

520[8] M. Clifford, J.E. Brown, in: Ø.M. Anderson, K.R. Markham (Eds), Flavonoids:

521 Chemistry, Biochemistry and Applications, CRC Press, Boca Raton, 2006, pp. 319-

$522 \quad 370$.

523[9] B. Holst, G. Williamson, Curr. Opin. Biotechnol. 18 (2008) 73-82.

524[10] I. Hernández, L. Alegre, F. Van Breusegem, S. Munné-Bosch, Trends Plant Sci. 14

$525 \quad$ (2009) 125-132.

526[11] I. Mueller-Harvey, J. Sci. Food Agric. 86 (2006) 2010-2037.

527[12] J.D. Lambert, J. Hong, G.-Y. Yang, J. Liao, C.S. Yang, Am. J. Clin. Nutr. 81 (suppl.)

$528 \quad$ (2005) 284S-291S.

529[13] W. Feucht, H. Dithmar, J. Polster, Internat. J. Mol. Sci. 8 (2007) 635-650. 
530[14] W. Feucht, D. Treutter, H. Dithmar, J. Polster, Tree Physiol. 28 (2008) 1783-1791.

531[15] W. Feucht, H. Dithmar, J. Polster, J. Bot. (2009) Article ID 842869 (doi:

$532 \quad 10.1155 / 2009 / 842869)$

533[16] J. Polster, H. Dithmar, W. Feucht, Biol. Chem. 384 (2003) 997-1006.

534[17] J. Bauer, K. Neubauer, H. Dithmar, J. Polster, W. Feucht, Adv. Food Sci. 31 (2009)

$535 \quad 82-88$.

536[18] J. Polster, H. Dithmar, R. Burgemeister, G. Friedemann, W. Feucht, Physiol. Plant.

$537 \quad 128$ (2006) 163-174.

538[19] D. Treutter, J. Chromatogr. 467 (1989) 185-193.

539[20] A.-P. Nifli, P.A. Theodoropoulos, S. Munier, C. Castagnino, E. Roussakis, H.E.

540 Katerinopoulos, J. Vercauteren, E. Castanas, J. Agric. Food Chem. 55 (2007) 2873-

5412878.

542[21] K. Suhling, P.M. French, D. Phillips, Photochem. Photobiol. Sci. 4 (2005) 13-22.

543[22] E.B. van Munster, T.W.J. Gadella, Adv. Biochem. Eng. Biotechnol. 95 (2005) 143-

$544 \quad 175$.

545[23] S.W. Botchway, A.W. Parker, R.H. Bisby, A.G. Crisostomo, Microsc. Res. Tech. 71

$546 \quad$ (2008) 267-273.

547[24] A. Osterrieder, C.M. Carvalho, M. Latijnhouwers, J.N. Johansen, C. Stubbs, S.

548 Botchway, C. Hawes, Traffic. 10 (2009) 1-13.

549[25] R.H. Bisby, S.W. Botchway, A.G. Crisostomo, J. Karolin, A.W. Parker, L. Schröder,

$550 \quad$ Spectroscopy 24 (2010) 137-142.

551[26] S.W. Botchway, M. Charnley, J.W. Haycock, A.W. Parker, D.L. Rochester, J.A.

552 Weinstein, J.A.G. Williams, PNAS, 105 (2008) 16071-16076.

553[27] P.T.C. So, C.Y. Dong, B.R. Masters, K.M. Berland, Annu. Rev. Biomed. Eng. 2

$554 \quad$ (2000) 399-429.

555[28] I. Sparkes, N. Tolley, I. Aller, J. Svozil, A. Osterrieder, S. Botchway, C. Mueller, L.

556 Frigerio, C. Hawes, Plant Cell 22 (2010) 1333-1343.

557[29] A. Rajavelu, Z. Tulyasheva, R. Jaiswal, A. Jeltsch, N. Kuhnert, BMC Biochemistry

558 12:16 (2011) Doi:10.1186/1471-2091-12-16.

559[30] W. Feucht, J. Polster, Z. Naturforsch. 56c (2001) 479-481. 
560[31] J.R. Lakowicz, Principles of Fluorescence Spectroscopy, fourth ed., Springer, New

$561 \quad$ York, 2006.

562[32] D.F. Eaton, Pure Appl. Chem. 60 (1988) 1107-1114.

563[33] Website: http://omlc.ogi.edu/spectra/PhotochemCAD/html/tryptophan.html

564[34] S. Sang, M.-J. Lee, Z. Hou, C.-T. Ho, C.S. Yang, J. Agric. Food Chem. 53 (2005)

565 9478-9484.

566[35] N.P. Slabbert, Tetrahedron 33 (1977) 821-824.

567[36] M.A. Soobrattee, V.S. Neergheen, A. Luximon-Ramma, O.I. Aruoma, T. Bahorun,

$568 \quad$ Mutation Res. 579 (2005) 200-213.

569[37] A.V. Probst, P.F. Fransz, J. Pazkowski, O. Mittelsten-Scheid, Plant J. 33 (2003) 743-

570749.

571[38] D. Hernandez-Verdun, J. Cell Sci. 99 (1991) 465-471.

572[39] F.L. Tobiason, R.W. Hemingway, G. Vergoten, Basic Life Sci. 66 (1999) 527-544.

573[40] K.O., Chu, C.C. Wang, C.Y. Chu, K.W. Choy, C.P. Pang, M.S. Rogers, Hum.

$574 \quad$ Reprod. 22 (2007) 280-287.

575[41] C.D. Kanakis, P.A. Tarantilis, M.G. Polissiou, S. Diamantoglou, H.A. Tajmir-Riahi,

$576 \quad$ Cell Biochem. Biophys. 49 (2007) 29-36.

577[42] W. Feucht, H. Dithmar, J. Polster, Plant Biol. 6 (2004) 696-701.

578[43] M.B. Inoue, M. Inoue, Q. Fernando, S. Valcic, B.N. Timmermann, J. Inorg.

$579 \quad$ Biochem. 88 (2002) 7-13.

580[44] W.A. Peer, D.E. Brown, B.W. Tague, G.K. Muday, L. Taiz, A.S. Murphy, Plant

$581 \quad$ Physiol. 126 (2001) 536-548.

582[45] D.E. Saslowsky, U. Warek, B.S.J. Winkel, J. Biol. Chem. 280 (2005) 23735-23740.

583[46] H.O. Gutzeit, Y. Henker, B. Kind, A. Franz, Biochem. Biophys. Res. Commun. 318

$584 \quad$ (2004) 490-495.

585[47] J. Grandmaison, R. Ibrahim, J. Plant Physiol. 147 (1996) 653-660.

586[48] M.Z. Fang, Y. Wang, N. Ai, Z. Hou, Y. Sun, H. Lu, W. Welsh, C.S. Yang, Cancer

587 Res. 63 (2003) 7563-7570.

588[49] T.M. Ehrman, D.J. Barlow, P.J. Hyland, J. Chem. Inf. Model 47 (2007) 254-263.

589[50] K.-C. Choi, M.G. Jung, Y.-H. Lee, J.C. Yoon, S.H. Kwon, H.-B. Kang, M.-J. Kim,

590 J.-H. Cha, Y.J. Kim, W.J. Jun, J.M. Lee, H.-G. Yoon, Cancer Res. 69 (2009) 583-

591592.

592[51] M. Glei, B.L. Pool-Zobel, Toxicol. in Vitro 20 (2005) 295-300. 
593[52] L. Guo, L.H. Wang, B. Sun, J.Y. Yang, Y.Q. Zhao, Y.X. Dong, M.I. Spranger, C.F.

$594 \quad$ Wu, J. Agric. Food Chem. 55 (2007) 5881-5891.

595[53] H. Yamada, H. Sugimura, T. Tsuneyoshi, J. Food Agric. Environ. 3 (2005) 73-76.

596[54] J. Ordovás, C.E. Smith, Nature Rev. Cardiol. 7 (2010) 510-519.

597[55] J. Zhao, Y. Pang, R.A. Dixon, Plant Physiol. 153 (2010) 437-443. 


\section{Legend to Figures}

599

\section{Fig. 1:}

601Structures of three flavanols, catechin (1), epicatechin (2), and epigallocatechin gallate (3), 602and one flavonol, quercetin (4) (note: A, B, C denote the flavonoid rings).

603

\section{Fig. 2:}

605The Jablonski diagramme depicting the energy levels of a molecule. $\mathrm{S}_{\mathrm{o}}$ represents the 606ground singlet states, $\mathrm{S}_{1}, \mathrm{~S}_{2}$ the excited singlet states; $\mathrm{T}_{1}$ the triplet excited states. Electronic 607levels are subdivided into vibrational levels $\left(v_{1}, v_{2} \ldots v_{n}\right)$. IC indicates internal conversion, $608 \mathrm{k}_{\text {fluorescence: }}$ rate of fluorescence leading from $S_{1}\left(v_{1}=0\right)$ to $S_{o}\left(v_{1}\right.$ or $\left.v_{n}\right), k_{I V R}$ intramolecular 609vibrational relaxation, $\mathrm{k}_{\mathrm{ISc}}$ : rate of intersystem crossing and $\mathrm{k}_{\text {quench }}$ : rate of reaction with 610other molecules, chemical or energy transfer.

\section{1}

\section{Fig. 3:}

613Time course of fluorescence lifetimes (ns) of catechin (2 mM) in $0.1 \mathrm{M}$ phosphate buffer in 614air or nitrogen atmospheres at pH 5.8, 7.1 and $8.2\left(\lambda_{\mathrm{ex}}=585 \mathrm{~nm}\right)$.

615

\section{Fig. 4:}

617Fluorescence decay curves of catechin, epicatechin and epigallocatechin gallate (EGCG) 618solutions (2 mM) in $0.1 \mathrm{M}$ phosphate buffer at $\mathrm{pH} 8.1\left(\lambda_{\mathrm{ex}}=585 \mathrm{~nm}\right)$.

\section{Fig. 5:}


621Fluorescence lifetime images $\left(\lambda_{\mathrm{ex}}=630 \mathrm{~nm}\right)$ of a cell from an onion epidermis soaked in 1 $622 \mathrm{mM}$ aqueous solutions of a) catechin ( $\left.\left.\tau_{1}=0.4 \mathrm{~ns}(82 \%), \mathrm{\tau}_{2}=2.6 \mathrm{~ns}(18 \%)\right), \mathrm{b}\right)$ 623epigallocatechin gallate ( $\tau=0.2 \mathrm{~ns})$ and $\mathrm{c}$ ) control in water without added flavanol. Image 624(A) shows a steady state image of the total emission lifetimes and image (B) shows the 625analysed fluorescence excited state map. The distribution of fluorescence lifetimes in (B) is 626illustrated in image (C), where the vertical axis represents the frequency and the horizontal 627axis represents lifetime in pico-seconds. (Note: control nucleus shows hardly any 628fluorescence in Fig. 5c).

629

630Supporting information

631Additional Supporting Information may be found in the online version of this article:

632Supplementary Table: Fluorescence lifetimes (ns) of flavanols in the presence of different 633histones (Tris buffers, pH 7 and 8). Pre-exponential factors are shown in brackets.

634

635Fig. S1. UV-Vis spectra of catechin, epicatechin and epigallocatechin gallate recorded from 636200 to $595 \mathrm{~nm}$.

637

638Video Clip S1. 3D stack of multiphoton excited $\left(\lambda_{\text {ex }}=630 \mathrm{~nm}\right)$ fluorescence image from an 639onion cell epidermis soaked in a $1 \mathrm{mM}$ aqueous solution of epigallocatechin gallate. Images 640were recorded at $0.5-2.0 \mu \mathrm{m}$ slices.

641 
642Please note: Wiley Blackwell are not responsible for the content or functionality of any 643supporting materials supplied by the authors. Any queries (other than missing material) 644should be directed to the corresponding author for the article.

645

646 


\section{Table 1}

648Fluorescence quantum yields of catechin, epicatechin and epigallocatechin gallate (EGCG) 649in methanol at $37^{\circ} \mathrm{C}$.

650

\begin{tabular}{ll}
\hline Compound & Quantum yield $^{\mathbf{a}}$ \\
\hline Catechin & 0.018 \\
Epicatechin & 0.015 \\
EGCG & 0.003 \\
\hline
\end{tabular}

651

$652^{\mathrm{a}}$ Estimated accuracy $= \pm 16 \%$

653 


\section{Table 2}

655Fluorescence lifetimes (ns) and pre-exponential factors ( $\mathrm{A}_{1}$ and $\left.\mathrm{A}_{2}\right)$ of externally added 656flavanols, which were absorbed by onion epidermis, and endogenous flavanols in Taxus 657baccata male cones ( \pm standard deviations).

658

\begin{tabular}{ccccc}
\hline Sample & $\mathbf{T}_{\mathbf{1}}$ (ns) & $\mathbf{A}_{\mathbf{1}} \mathbf{\%}$ & $\mathbf{T}_{\mathbf{2}}$ (ns) & $\mathbf{A}_{\mathbf{2}} \mathbf{\%}$ \\
\hline $\begin{array}{c}\text { Onion epidermis: } \\
\text { control in water }\end{array}$ & $0.84^{\mathrm{a}}$ & & & \\
$\begin{array}{c}\text { Onion epidermis: } \\
\quad \text { + catechin }\end{array}$ & $0.5 \pm 0.04$ & $77.5 \pm 6.92$ & $2.7 \pm 0.19$ & $22.5 \pm 6.92$ \\
+ epigallocatechin gallate & $0.25 \pm 0.05$ & $99.2 \pm 1.13$ & & \\
$\begin{array}{c}\text { (EGCG) } \\
\text { Taxus baccata male cones: } \\
\text { in water }\end{array}$ & $0.2 \pm 0.03$ & $93.4 \pm 6.46$ & $0.5 \pm 0.2$ & $6.6 \pm 6.46$ \\
\hline
\end{tabular}

659

$660^{\mathrm{a}}$ The control had a very low photon count in the absence of externally added flavanols and 661the data were quite noisy (see Fig. 5c), therefore it was not possible to obtain a standard 662deviation of the background lifetime.

663

664 
665Figure 1:

666

667

668

669 
670Fig 2

671

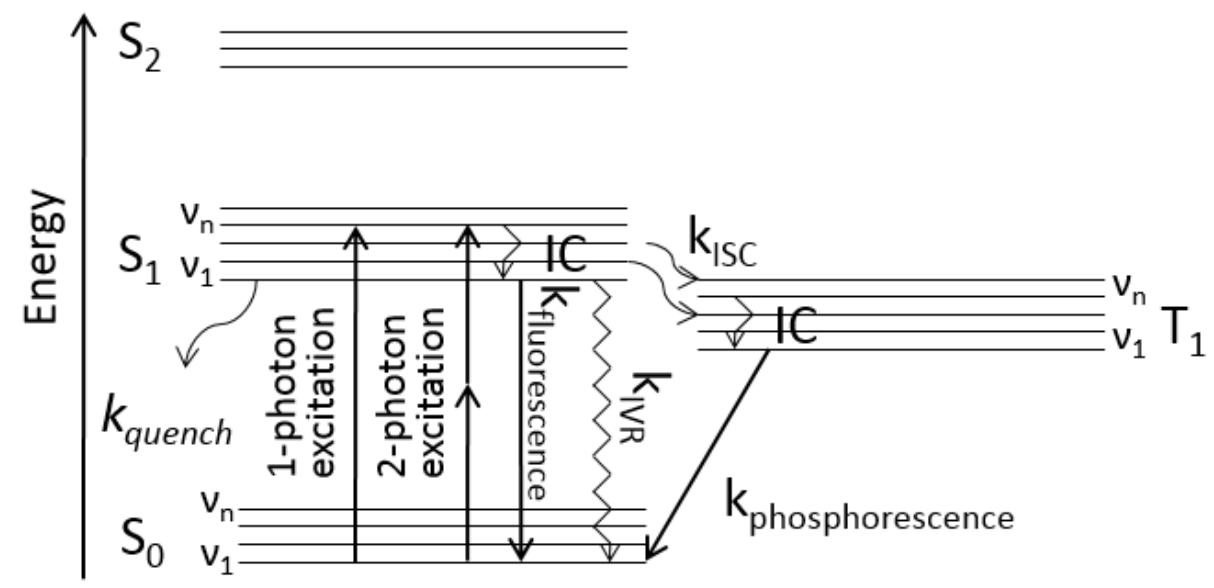

672

673

674Fig 3

675

676

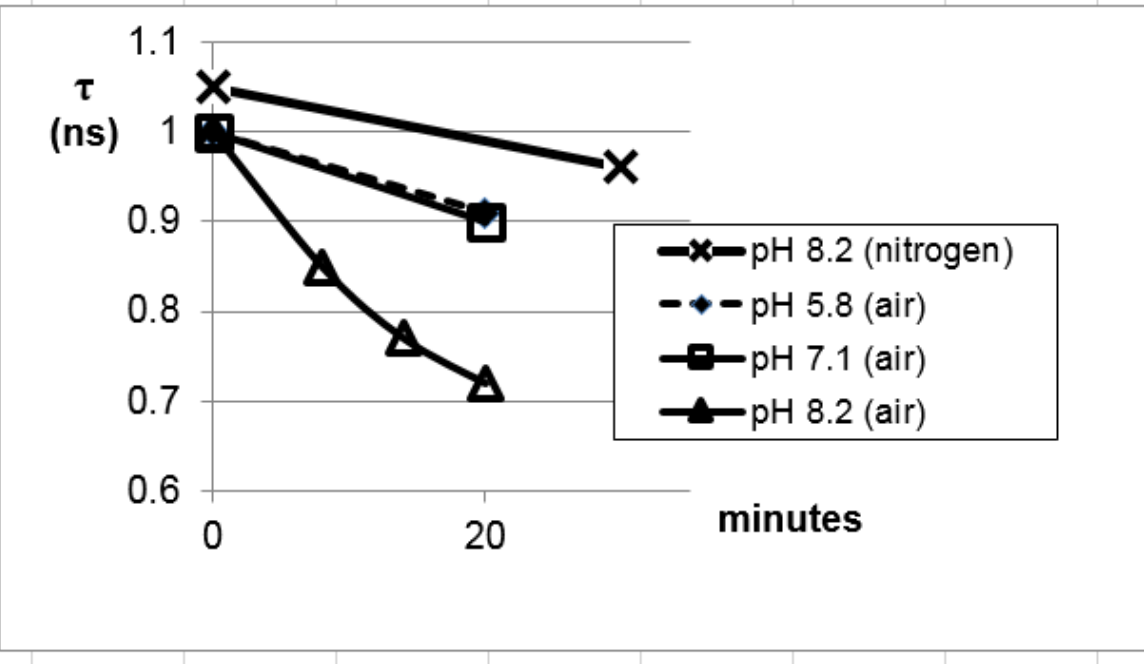

677 
678Fig 4

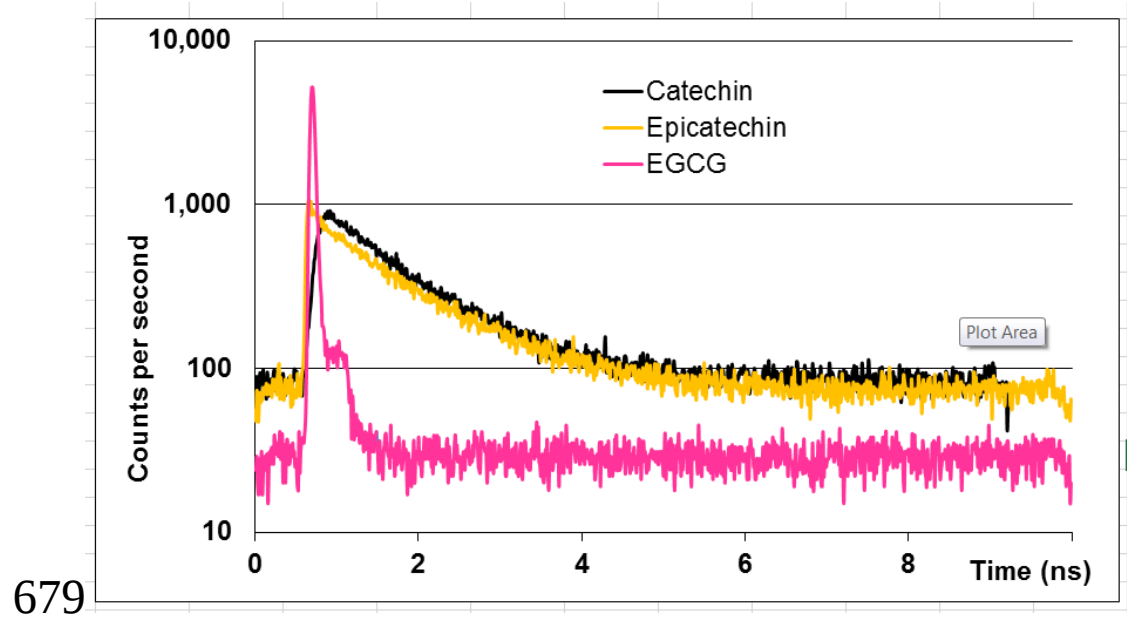

680 
681Fig 5a

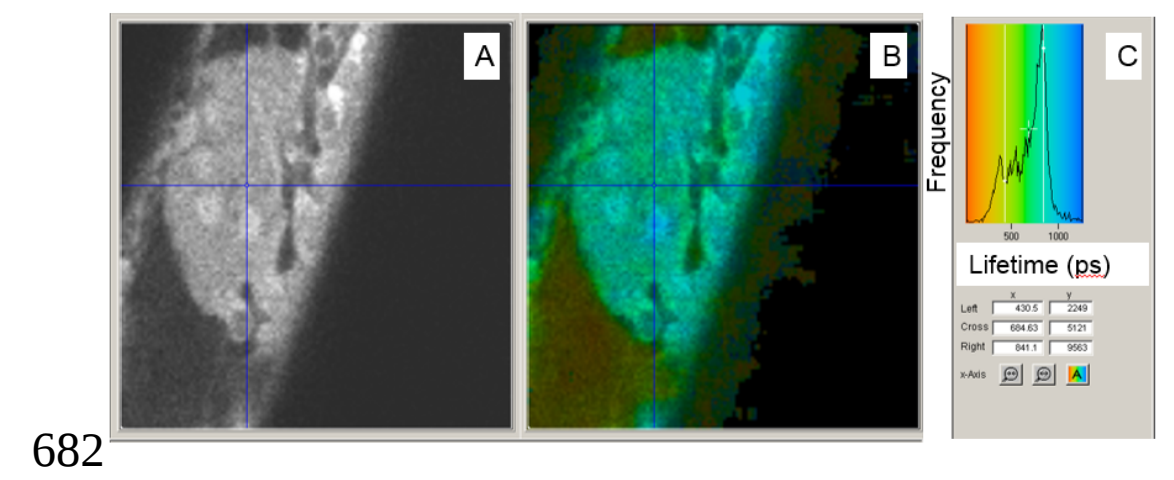

683Fig $5 \mathrm{~b}$

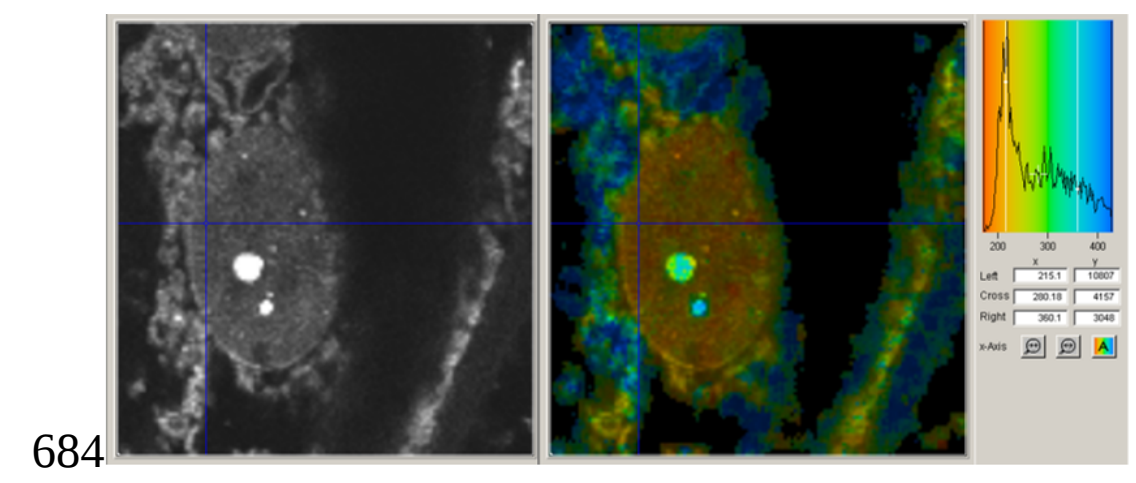

685Fig 5c

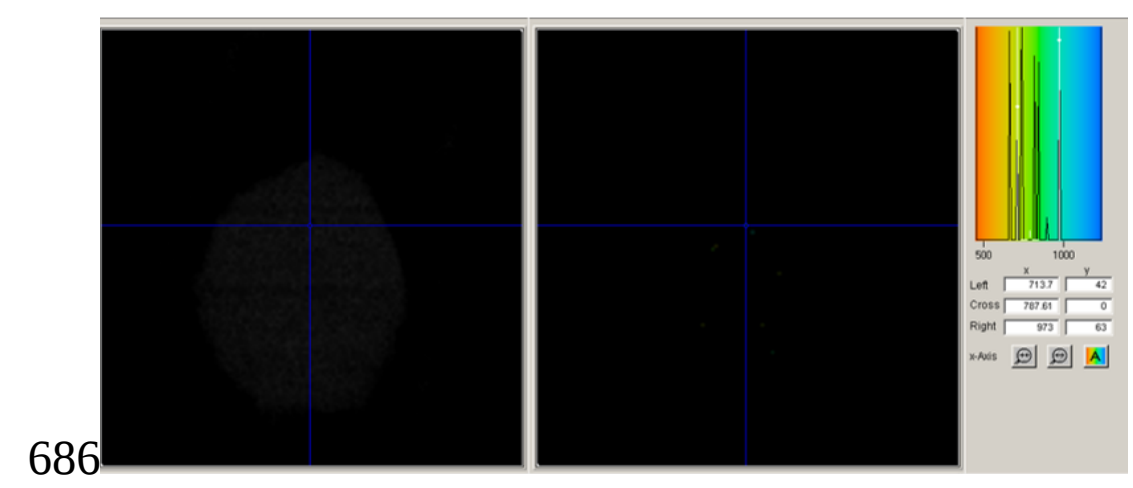

687

688 


\section{Fig S1}

690Figure S1: UV-Vis spectra of catechin, epicatechin and epigallocatechin 691gallate recorded from 200 to $595 \mathrm{~nm}$.

\section{2}

\section{Catechin}

\section{4}

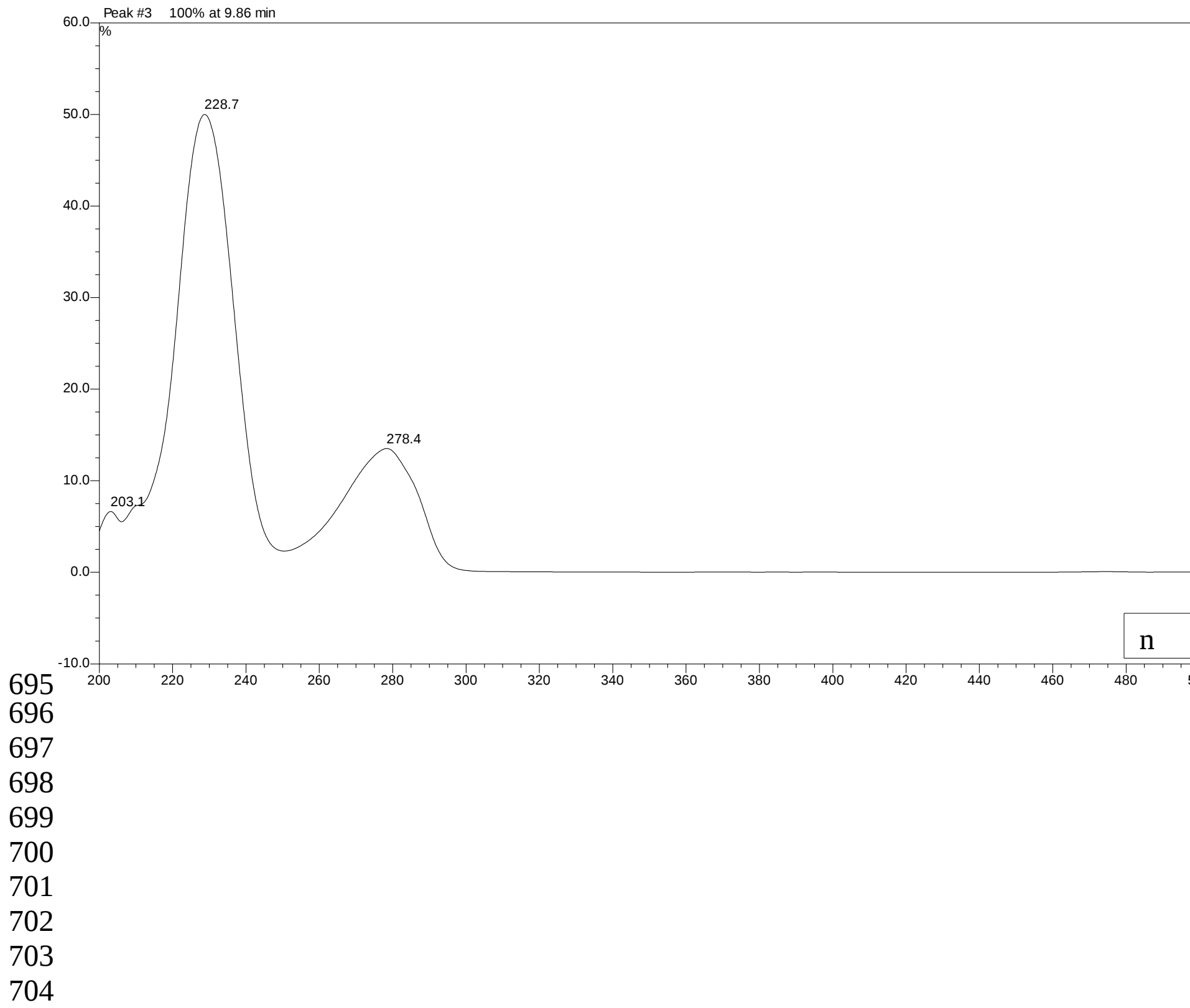




\section{Epicatechin}

706

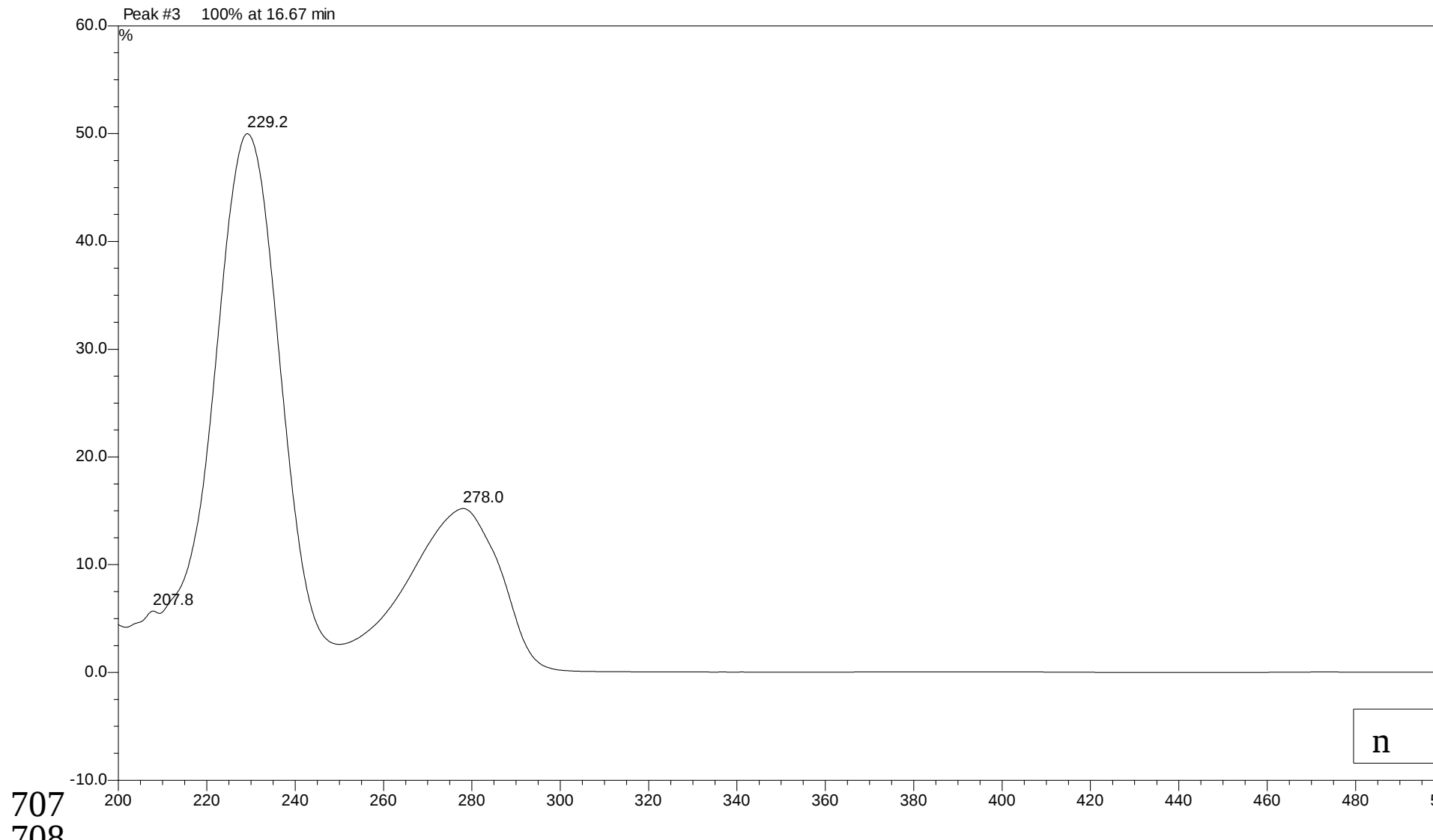

708

709

710

711

712

713

714

715

716 


\section{Epicatechin gallate}

718

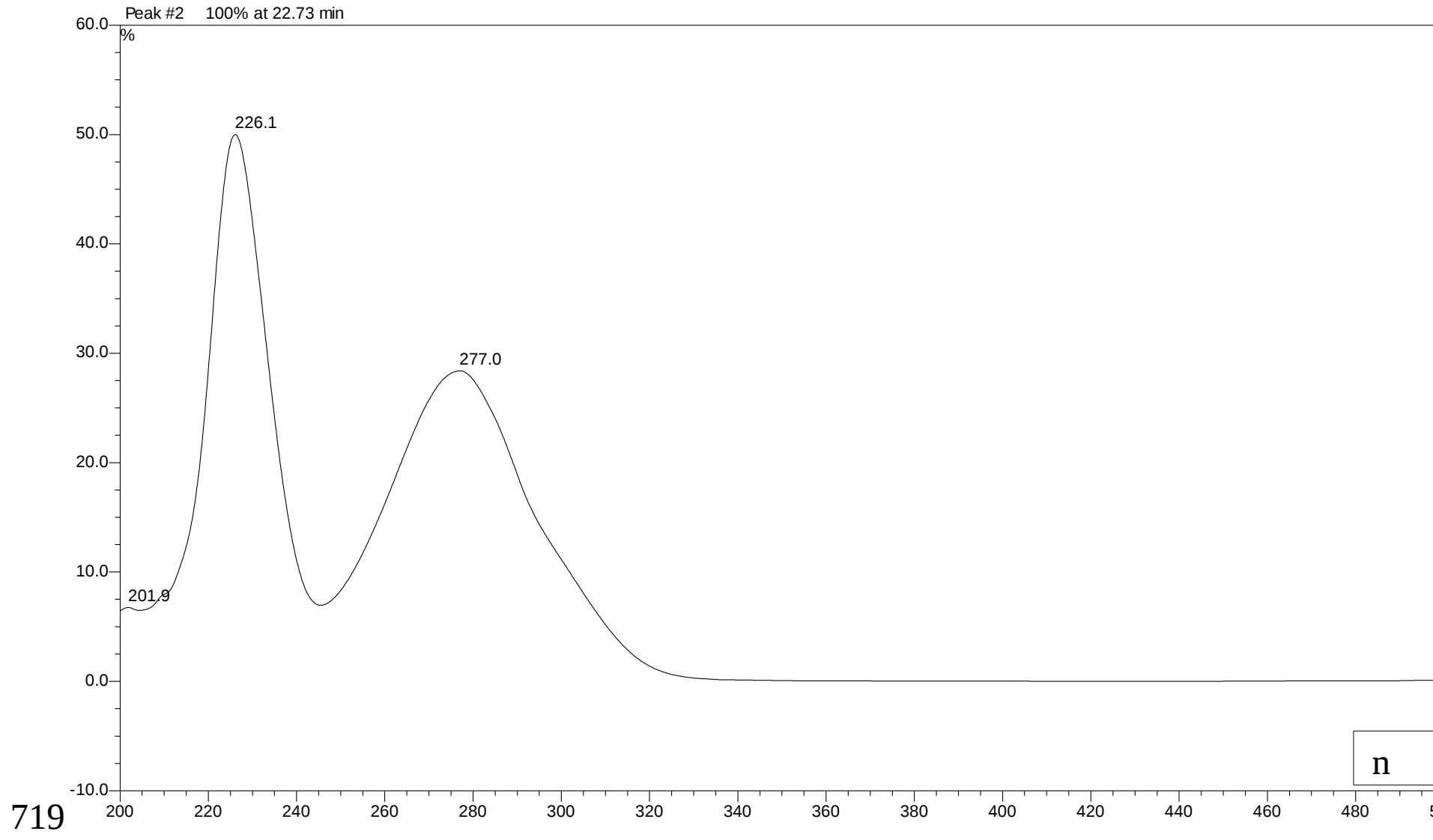




\section{Supplementary Table}

721Fluorescence lifetimes (ns) of flavanols in the presence of different histones (Tris buffers, pH 7 and 8). Pre-exponential factors

722are shown in brackets.

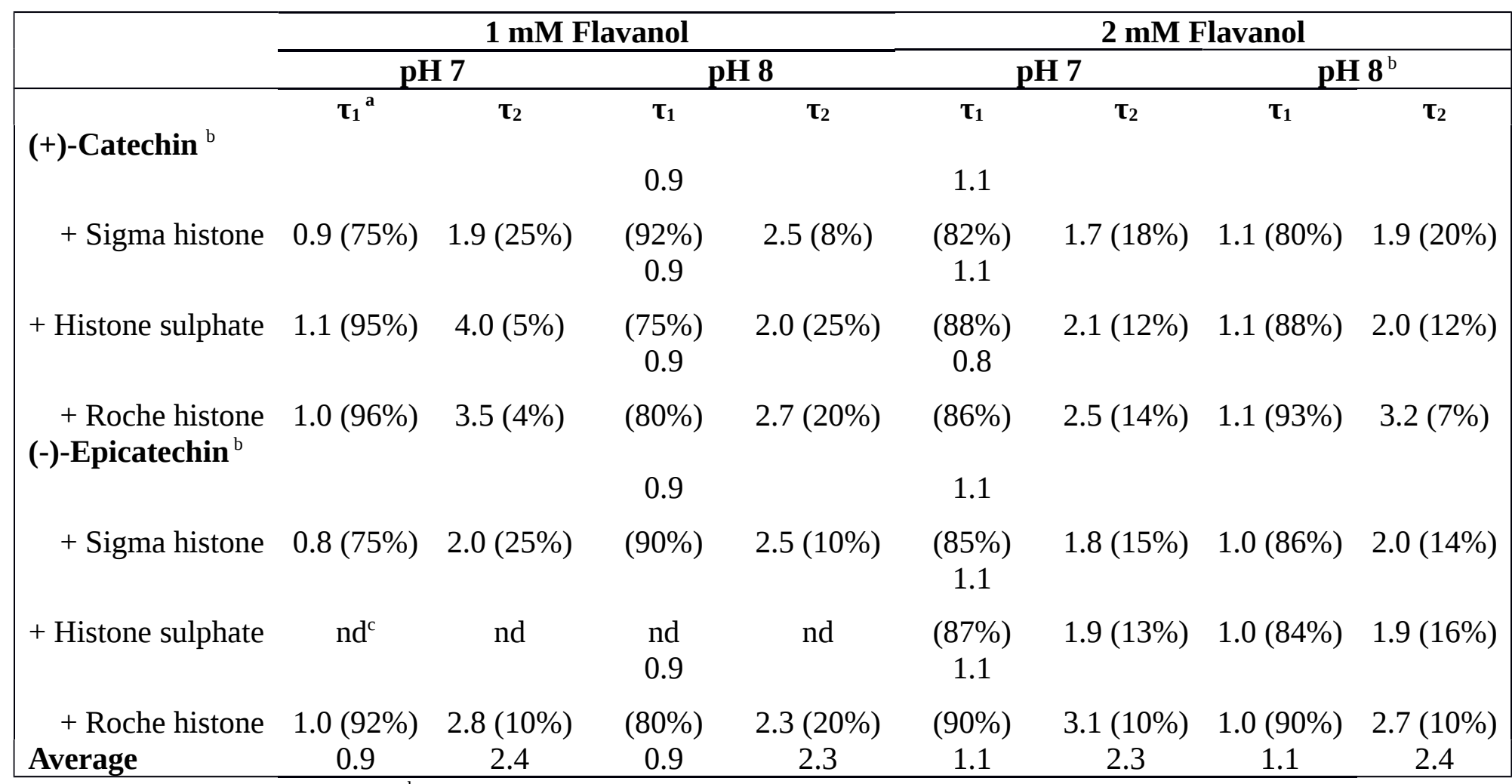

$723^{\mathrm{a}}$ Experimental error is $\pm 50 \mathrm{ps}$; ${ }^{\mathrm{b}}$ For comparison, lifetime measurements in $0.1 \mathrm{M}$ phosphate buffer at $\mathrm{pH} 8.1 \mathrm{gave} \mathrm{\tau}=1.0 \mathrm{~ns}$ for

724catechin and $\tau_{1}=1.1(72 \%)$ and $\tau_{2}=0.1 \mathrm{~ns}(28 \%)$ for epicatechin (note: Due to the poor signal-to-noise at longer times the errors 
725are significantly larger for the second lifetime $\left(\tau_{2}\right)$ and the pre-exponential factors of less than $10 \%$ may be due to a fluorescence 726contribution from impurities); ${ }^{\mathrm{c}}$ nd $=$ not determined. 